\title{
On Fuglede's conjecture and the existence of universal spectra
}

\author{
Bálint Farkas, Máté Matolcsi, Péter Móra \\ February 2, 2008
}

\begin{abstract}
Recent methods developed by Tao [17], Kolountzakis and Matolcsi [7] have led to counterexamples to Fugelde's Spectral Set Conjecture in both directions. Namely, in $\mathbb{R}^{5}$ Tao produced a spectral set which is not a tile, while Kolountzakis and Matolcsi showed an example of a non-spectral tile. In search of lower dimensional nonspectral tiles we were led to investigate the Universal Spectrum Conjecture (USC) of Lagarias and Wang [13]. In particular, we prove here that the USC and the "tile $\rightarrow$ spectral" direction of Fuglede's conjecture are equivalent in any dimensions. Also, we show by an example that the sufficient condition of Lagarias and Szabó [12] for the existence of universal spectra is not necessary. This fact causes considerable difficulties in producing lower dimensional examples of tiles which have no spectra. We overcome these difficulties by invoking some ideas of Révész and Farkas [2], and obtain non-spectral tiles in $\mathbb{R}^{3}$.

Fuglede's conjecture and the Universal Spectrum Conjecture remains open in 1 and 2 dimensions. The 1 dimensional case is closely related to a number theoretical conjecture on tilings by Coven and Meyerowitz [1].
\end{abstract}

2000 Mathematics Subject Classification. Primary 52C22, Secondary 20K01, $42 \mathrm{~B} 99$.

Keywords and phrases. Translational tiles, spectral sets, Fuglede's conjecture, universal spectrum.

\section{Introduction}

We briefly summarize the appearing concepts and some related known results. Let $G$ denote some finite Abelian group or $\mathbb{Z}^{d}$ or $\mathbb{R}^{d}$. (The notions below can be extended to the more general setting of locally compact Abelian groups, but we will restrict our attention to the mentioned cases.) We will always consider the standard Haar measures on these groups. The cyclic group of $n$ elements will be denoted by $\mathbb{Z}_{n}$. The dimension of a finite Abelian group $G$ is understood to be the smallest $d$ such that $G$ is a factor group of $\mathbb{Z}^{d}$. The Fourier zero-set $Z_{T}$ of a set $T \subset G$ is defined as the zero-set of the Fourier transform of the indicator function of $T$, i.e., $Z_{T}:=\left\{v \in \hat{G}: \hat{\chi}_{T}(v)=0\right\}$; its complement is denoted by $Z_{T}^{c}:=\hat{G} \backslash Z_{T}$. 
Definition. A set $\Lambda \subseteq \hat{G}$ is called a spectrum of a bounded open set $T \subseteq G$ if the restricted characters $\left\{\left.\lambda(z)\right|_{z \in T}\right\}_{\lambda \in \Lambda}$ form a complete orthogonal system in $L_{2}(T)$. A bounded open set $T \subseteq G$ is called spectral if it possesses a spectrum.

It is immediate from the definition that for a subset $T$ of a finite group $G, \Lambda \subset \hat{G}$ is a spectrum of $T$ if and only if $\Lambda-\Lambda \subset Z_{T} \cup\{0\}$.

The other class of sets under study is that of translational tiles:

Definition. A bounded open subset $T$ of $G$ is said to be a tile if the whole group $G$ can be covered by translated disjoint copies of $T$ up to a set of zero measure. That is, there exists a set $T^{\prime} \subseteq G$, called a tiling complement of $T$ such that $T^{\prime}+T=\cup_{t^{\prime} \in T^{\prime}}\left(t^{\prime}+T\right)=G$ up to a set of zero measure, and the union is assumed to be disjoint, i.e., $T-T \cap T^{\prime}-T^{\prime}=\{0\}$.

In a finite group $G$ there are two well-known (easy) necessary and sufficient conditions for $T^{\prime}$ to be a tiling complement of $T$ : one is that $|T| \cdot\left|T^{\prime}\right|=|G|$ and $T-T \cap T^{\prime}-T^{\prime}=\{0\}$, while the other is that $|T| \cdot\left|T^{\prime}\right|=|G|$ and $Z_{T} \cup Z_{T^{\prime}}=\hat{G} \backslash\{0\}$.

Connecting these two notions Fuglede's spectral set conjecture [3] asserts that a bounded open subset of $\mathbb{R}^{d}$ is a tile if and only if it is spectral.

Fuglede [3] proved the special case of the conjecture, when the spectrum or the tiling complement is assumed to be a lattice. Later on, for many years several positive results seemed to indicate the validity of the conjecture (see e.g. $[5,4,6,9,10,11,13,15]$. Recently, however, Tao [17] disproved the "spectral $\rightarrow$ tile" direction in $\mathbb{R}^{5}$ and higher dimensions. Kolountzakis and Matolcsi [8] reduced this dimension to 3, and also constructed a counterexample to the "tile $\rightarrow$ spectral" direction in $\mathbb{R}^{5}$, see [7]. Révész and Farkas refined the arguments of [7] to produce a non-spectral tile in $\mathbb{R}^{4}$. One of the aims of this paper is to remove the existing discrepancy between the dimensions by presenting an example of a tile in $\mathbb{R}^{3}$ which does not have a spectrum. In doing so, we will also investigate the relation of Fuglede's conjecture to the Universal Spectrum Conjecture of Lagarias and Wang [13] and a certain sufficient condition of Lagarias and Szabó [12] on the existence of universal spectra. The arguments are based on combinatorial and Fourier analytic conditions of tiling and spectrality but, unfortunately, some numerical calculations cannot be avoided. The methods developed in this paper may be useful later in 1 or 2 dimensional considerations. In fact, it is known [9] that in $\mathbb{Z}$ the "tile $\rightarrow$ spectral" direction of Fuglede's conjecture would follow form a number theoretical conjecture on tilings by Coven and Meyerowitz [1]. Therefore, any example of a non-spectral tile in $\mathbb{Z}$ would immediately disprove the Coven-Meyerowitz conjecture, too. However, despite some numerical experiments in 1-dimensional cyclic groups, we have not been able to find such examples yet.

First, we review and complement some relevant results from the literature. The general approach, developed first by Tao [17], is to find a counterexample in a finite Abelian group and then transfer the example to the lattice $\mathbb{Z}^{d}$ and, finally, to $\mathbb{R}^{d}$. We recall the following results:

Theorem 1 ([7]). Suppose $B \subseteq \mathbb{Z}^{d}$ is a finite set and $Q=(0,1)^{d}$ is the unit cube. Then $B$ is a spectral set in $\mathbb{Z}^{d}$ if and only if $B+Q$ is a spectral set in $\mathbb{R}^{d}$.

It is also clear that if $A$ tiles $\mathbb{Z}^{d}$ then $B+Q$ tiles $\mathbb{R}^{d}$. This means that any counterexample in $\mathbb{Z}^{d}$ to the "tile $\rightarrow$ spectral" direction of Fuglede's conjecture will automatically lead to a counterexample in the corresponding Euclidean space $\mathbb{R}^{d}$. 
Theorem $2([7])$. Let $\mathbf{n}=\left(n_{1}, \ldots, n_{d}\right) \in \mathbb{N}^{d}$ and consider a set $A \subseteq G=\mathbb{Z}_{n_{1}} \times \cdots \times \mathbb{Z}_{n_{d}}$. For the set

$$
T=T(\mathbf{n}, k)=\left\{0, n_{1}, 2 n_{1}, \ldots,(k-1) n_{1}\right\} \times \cdots \times\left\{0, n_{d}, 2 n_{d}, \ldots,(k-1) n_{d}\right\}
$$

define $B(k)=A+T$. Then, for large enough values of $k$, the set $B(k) \subset \mathbb{Z}^{d}$ is spectral in $\mathbb{Z}^{d}$ if and only if $A$ is spectral in $G$.

It is also clear that if $A$ tiles $G$ then $B(k)$ tiles $\mathbb{Z}^{d}$ for all values of $k$. This means that any counterexample in a finite group to the "tile $\rightarrow$ spectral" direction of Fuglede's conjecture will automatically lead to a counterexample in the corresponding lattice $\mathbb{Z}^{d}$. Therefore, our task is to construct a non-spectral tile in some 3-dimensional finite group.

Remark 1. The corresponding results also hold in this generality in the other direction of Fuglede's conjecture. Namely, any spectral set in $\mathbb{Z}^{d}$ which is not a tile leads (by addition of the unit cube) to a spectral set in $\mathbb{R}^{d}$ which is not a tile. Also, any spectral set in a finite group $G=\mathbb{Z}_{n_{1}} \times \cdots \times \mathbb{Z}_{n_{d}}$ which is not a tile leads (by constructing $B(k)$ as above) to a spectral set in $\mathbb{Z}^{d}$ which is not a tile. The proof of the first statement is fairly straightforward, while the second is essentially contained in [17] and also in [14], Proposition 2.1 and 2.5. We will not need these statements here, but it is instructive to see that in both directions of Fuglede's conjecture any finite group counterexample can be transferred automatically to the Euclidean setting, where the conjecture was originally formulated.

\section{The equivalence of the Universal Spectrum Con- jecture and Fuglede's conjecture}

In order to construct a non-spectral tile in a 3-dimensional finite group we will need the notion of universal spectrum (see [13]). This notion was originally defined in the Euclidean setting [13], but all known sufficient conditions for the existence of universal spectra go back to considerations in finite groups $[13,12]$. Therefore, in this paper, for the sake of simplicity we will remain in the finite setting, which will suffice for our purposes.

Definition. A subset $S \subset \hat{G}$ is a universal spectrum of a set $T \subset G=\mathbb{Z}_{n_{1}} \times \cdots \times \mathbb{Z}_{n_{d}}$ if $S$ is a spectrum of all tiling complements $T^{\prime}$ of $T$ in $G$.

The Universal Spectrum Conjecture (USC) of Lagarias and Wang stated that in any finite Abelian group $G$ all tiles $T$ posses a universal spectrum. This conjecture was first disproved in $[7]$ in $\mathbb{Z}_{6}^{5}$, and later in $[2]$ in $\mathbb{Z}_{6}^{4}$, as an essential step in producing non-spectral tiles in $\mathbb{R}^{5}$ and $\mathbb{R}^{4}$, respectively. We now prove that the USC and the "tile $\rightarrow$ spectral" direction of Fuglede's conjecture are equivalent in the sense that the failure of one in any dimension will automatically result in the failure of the other in the same dimension (i.e., it is not by chance that the examples of [7] and [2] led to counterexamples to Fuglede's conjecture).

Proposition 3. For any dimension d, the Universal Spectrum Conjecture is valid for all $d$-dimensional finite groups if and only if all tiles are spectral sets in all d-dimensional finite groups. 
Proof. One direction of this statement is trivial. Namely, if $T$ is a non-spectral tile in a group $G$ then any tiling complement $T^{\prime}$ does not possess a universal spectrum in $\hat{G}$.

Conversely, assume that we find a $d$-dimensional group $G=\mathbb{Z}_{n_{1}} \times \cdots \times \mathbb{Z}_{n_{d}}$ and a tile $T \subset G$ which does not have a universal spectrum. Let $k:=|T|$ and $n:=|G|$. We will exhibit a non-spectral tile $R$ in a larger (but still $d$-dimensional) group $G_{1}:=$ $\mathbb{Z}_{n_{1}} \times \cdots \times \mathbb{Z}_{n_{d}} \times \mathbb{Z}_{p}$, where $p$ is a large integer, relatively prime to $n_{1}, \ldots, n_{d}$.

Note that $S \subset \hat{G}$ is a universal spectrum of $T$ if and only if $|S|=n / k$ and $S-S \subset$ $\cap_{j=1}^{m} Z_{T_{j}^{\prime}} \cup\{0\}$, where $T_{j}^{\prime}$ run through all possible tiling complements of $T$. By assumption $T$ does not have a universal spectrum, which implies that for any set $S \subset \hat{G},|S|=n / k$ we have a "witness" $\mathbf{v}_{S} \in S-S$ such that $\mathbf{v}_{S} \notin \cap_{j} Z_{T_{j}^{\prime}} \cup\{0\}$. Let $\mathbf{v}_{1}, \mathbf{v}_{2}, \ldots, \mathbf{v}_{r}$ denote the finite set of all such witnesses. Consider now the matrix

$$
A=\left(\begin{array}{cccc}
\hat{\chi}_{T_{1}^{\prime}}\left(\mathbf{v}_{1}\right) & \hat{\chi}_{T_{2}^{\prime}}\left(\mathbf{v}_{1}\right) & \cdots & \hat{\chi}_{T_{m}^{\prime}}\left(\mathbf{v}_{1}\right) \\
\hat{\chi}_{T_{1}^{\prime}}\left(\mathbf{v}_{2}\right) & \hat{\chi}_{T_{2}^{\prime}}\left(\mathbf{v}_{2}\right) & \cdots & \hat{\chi}_{T_{m}^{\prime}}\left(\mathbf{v}_{2}\right) \\
\vdots & & \ddots & \vdots \\
\hat{\chi}_{T_{1}^{\prime}}\left(\mathbf{v}_{r}\right) & \hat{\chi}_{T_{2}^{\prime}}\left(\mathbf{v}_{r}\right) & \cdots & \hat{\chi}_{T_{m}^{\prime}}\left(\mathbf{v}_{r}\right)
\end{array}\right) .
$$

We know that each row contains a non-zero entry. We now choose an integer vector $\mathbf{k}:=\left(k_{1}, k_{2}, \ldots, k_{m}\right)^{\top}$ such that $A \mathbf{k} \neq 0$ and $k_{1}+k_{2}+\cdots+k_{m}=p$ is relatively prime to $n_{1}, n_{2}, \ldots, n_{d}$. (It is easy to see that such choice is possible, as the $A \mathbf{k} \neq 0$ condition means only an exclusion of $r$ hyperplanes, and the relative prime condition means only an exclusion of a set of density strictly less than 1.)

We will now glue together the desired non-spectral tile $R \subset G_{1}$ from several copies of the sets $T_{1}^{\prime}, \ldots, T_{m}^{\prime}$. The idea is that we can consider $G_{1}$ as $p$ "layers" of $G$ and we will copy the sets $T_{j}^{\prime}$ on different layers.

We can regard the elements of $G_{1}$ as column vectors of length $d+1$. (Note, however, that the dimension of $G_{1}$ is still $d$ as $p$ was chosen relatively prime to $n_{1}, \ldots, n_{d}$; in fact it would suffice that $p$ is relatively prime to one them.) Also, the elements of $\hat{G}$ can be regarded as row vectors, the action of a character $\gamma \in \hat{G}$ on an element $\mathbf{x} \in G$ being defined as $\gamma(\mathbf{x}):=e^{\sum_{j=1}^{d+1} \gamma_{j} x_{j} / n_{j}}\left(\right.$ where $\left.n_{d+1}:=p\right)$. Let $\mathbf{z}_{j}=(0,0, \ldots, j)^{\top}$. For any set $A \subset G$ the notation $\tilde{A}$ will stand for the set $A$ extended by zero in the last coordinate. Let also $1=\sigma_{1} \leq \sigma_{2} \leq \cdots \leq \sigma_{p}=m$ be a sequence of integers, the number $i$ occurring exactly $k_{i}$ times among $\sigma_{j}$ (recall that $k_{1}+k_{2}+\cdots+k_{m}=p$ ). Consider the set

$$
R=\bigcup_{j=1}^{p}\left(\mathbf{z}_{j}+\tilde{T_{\sigma_{j}}^{\prime}}\right)
$$

We claim that $R$ is a tile in $G_{1}$ and it is not spectral. It is clear that $R$ tiles $G_{1}$ because a tiling complement can be given as $\tilde{T}$.

Consider any set $L \subset \hat{G}_{1},|L|=|R|$ as a candidate for being a spectrum of $R$. By the pigeonhole principle there exist an $L_{1} \subset L,\left|L_{1}\right|=n / k$ such that the last coordinates of the elements of $L_{1}$ are equal. Consider the set $\tilde{S}_{1}$ whose elements have the same coordinates as those of $L_{1}$ except for the last coordinate which is set to 0 in $\tilde{S}_{1}$. Then $\tilde{S}_{1}-\tilde{S}_{1}=L_{1}-L_{1} \subset L-L$. Consider now the witness $\mathbf{v}_{S_{1}}$ corresponding to $S_{1}$, and the extended vector $\tilde{\mathbf{v}_{S_{1}}}$. We have

$$
\hat{\chi}_{R}\left(\tilde{\mathbf{v}_{S_{1}}}\right)=k_{1} \cdot \hat{\chi}_{T_{1}^{\prime}}\left(\mathbf{v}_{S_{1}}\right)+k_{2} \cdot \hat{\chi}_{T_{2}^{\prime}}\left(\mathbf{v}_{S_{1}}\right)+\cdots+k_{m} \cdot \hat{\chi}_{T_{m}^{\prime}}\left(\mathbf{v}_{S_{1}}\right) \neq 0
$$


by construction. This shows that $R$ is not spectral in $G_{1}$ and the proof is complete.

Remark 2. One can also introduce the notion of universal tiling complement. A set $U \subset \hat{G}$ is a universal tiling complement of $T \subset G$ if $U$ is a tiling complement in $\hat{G}$ of all spectra of $T$.

Then one can prove the "dual" of the statement above, i.e., that all spectral sets are tiles in all $d$-dimensional finite groups if and only if all spectral sets possess universal tiling complements in all $d$-dimensional finite groups. In fact, one can use an analogous construction as above, building up layer by layer a spectral set which is not a tile in a larger group $G_{1}=G \times \mathbb{Z}_{p}$ (to see that the constructed set does not tile $G_{1}$ one needs to recall the Fourier condition $Z_{T} \cup Z_{T^{\prime}}=G \backslash\{0\}$ of tiling pairs). We do not give a detailed proof here as we will not need this result. However, it is very well possible that this statement can be useful in producing 1 or 2 dimensional examples in the future.

\section{A 3-dimensional tile without spectrum}

The results of the previous section show that our task is reduced to finding a tile $T$ of a 3-dimensional finite group $G$ which does not have a universal spectrum. We will exhibit such a set in $\mathbb{Z}_{24}^{3}$. Unfortunately, it is not at all straightforward to check whether a set $T$ possesses universal spectra or not. There is an elegant sufficient condition by Lagarias and Szabó [12]:

Proposition 4. For a given set $T$ in a finite group $G$, if a set $S \subset \hat{G}$ satisfies the conditions $|S|=|G| /|T|$ and $S-S \subset Z_{T}^{c}$ then $S$ is a universal spectrum of $T$, and also $S$ is a universal tiling complement of $T$.

Proof. We need to prove the second part of the proposition, which is not contained in [12]. Assume $L$ is any spectrum of $T$. Then $|L| \cdot|S|=\hat{G}$ and $L-L \cap S-S=\{0\}$, because $L-L \subset Z_{T} \cup\{0\}$ and $S-S \subset Z_{T}^{c}$. It follows that $L+S=\hat{G}$.

In fact, in [12] it is tentatively conjectured that the existence of such set $S$ is also a necessary condition for the existence of universal spectrum. If it were so, we could simply use the duality argument of [7] to produce a set without universal spectrum in $G=\mathbb{Z}_{24}^{3}$. The idea is to use the mod 8 log-Hadamard matrix $K$ given in [8] (i.e., the matrix with entries $e^{2 \pi i K_{j, k}}$ is a complex Hadamard matrix containing $8^{\text {th }}$ roots of unity):

$$
K:=\frac{1}{8}\left(\begin{array}{cccccc}
0 & 0 & 0 & 0 & 0 & 0 \\
0 & 4 & 2 & 6 & 6 & 2 \\
0 & 2 & 4 & 1 & 5 & 6 \\
0 & 6 & 3 & 4 & 2 & 7 \\
0 & 6 & 7 & 2 & 4 & 3 \\
0 & 2 & 6 & 5 & 1 & 4
\end{array}\right)
$$

Then, following a decomposition in [8], one can define a spectral set $T_{1}$ in $\mathbb{Z}_{24}^{3}$ with spectrum $L$ as (again the columns are the elements of $G$, while the rows correspond to 
elements of the dual group $\hat{G}$ )

$$
T_{1}:=\left(\begin{array}{cccccc}
0 & 2 & 4 & 1 & 5 & 6 \\
0 & 6 & 3 & 4 & 2 & 7 \\
0 & 6 & 7 & 2 & 4 & 3
\end{array}\right) \quad \text { and } \quad L:=3\left(\begin{array}{ccc}
0 & 0 & 0 \\
0 & 1 & 1 \\
1 & 0 & 0 \\
0 & 1 & 0 \\
0 & 0 & 1 \\
7 & 1 & 1
\end{array}\right) .
$$

Note that $24 K=L T_{1} \bmod 24$, therefore $L$ is indeed a spectrum of $T_{1}$ in $G$. Note also, that $L$ is contained in the subgroup of elements whose coordinates are all divisible by 3 . This subgroup has $8^{3}$ elements, hence $L$ cannot tile this subgroup due to obvious divisibility reasons. It is also well-known (and easy) that this implies that $L$ cannot tile $\hat{G}$ either.

It is not hard to see that $T_{1}$ tiles $G$ (this can be seen e.g. via the homomorphism $\varphi: G \rightarrow \mathbb{Z}_{24}$ induced by the row vector $(2,9,3)$, but we will need to modify $T_{1}$ later anyway), but the existence of a set $S \subset \hat{G},|S|=24^{3} / 6$ and $S-S \subset Z_{T_{1}}^{c}$ is impossible due to the following reason: such an $S$ would be a tiling complement of $L$ by Proposition 4, which is impossible as $L$ does not tile $\hat{G}$.

If the sufficient condition of Proposition 4 were also necessary then we could conclude that $T_{1}$ does not have a universal spectrum in $G$. We will show in the Appendix, however, by means of a particular example, that the condition of Proposition 4 is not necessary. Of course it still might happen that the set $T_{1}$ above does not have a universal spectrum but, in any case, we are unable to check it at the time of writing. (In general, even the elegant sufficient condition of Proposition 4 seems to be hard to check algorithmically in large groups, let alone finding all tiling complements of a given set.) The failure of the necessity of the Lagarias-Szabó condition poses some difficulty in checking whether a set possesses universal spectra, and therefore presents an obstacle to finding a 3-dimensional counterexample to Fuglede's conjecture. We will use ideas of Farkas and Révész to overcome this difficulty. The observation is that we are free to add +8 or +16 to the entries of $T_{1}$ without ruining the decomposition $24 K=L T_{1} \bmod 24$. We must find an alteration $T$ of $T_{1}$ such that the existence of a universal spectrum of $T$ can be excluded.

Proposition 5. The set

$$
T:=\left(\begin{array}{cccccc}
0 & 10 & 20 & 1 & 21 & 14 \\
0 & 22 & 3 & 20 & 2 & 7 \\
0 & 22 & 23 & 18 & 4 & 11
\end{array}\right)
$$

is a tile in $G=\mathbb{Z}_{24}^{3}$ which does not have a universal spectrum.

Proof. As observed before, the decomposition $24 K=L T$ still holds, therefore $L$ is a spectrum of $T$.

Consider all the $\bmod 24$ vectors $\mathbf{v}_{i j}:=\mathbf{l}_{i}-\mathbf{l}_{j} \in \hat{G}$ where $\mathbf{l}_{i}, \mathbf{l}_{j}$ are arbitrary rows of the matrix $L$. For each such vector $\mathbf{v}_{i j}$ we will exhibit a tiling complement $T_{i j}^{\prime}$ of $T$ in $G$ in such a way that $v_{i j} \notin Z_{T_{i j}^{\prime}}$. Accepting the existence of such $T_{i j}^{\prime}$ for the moment, we can easily show that $T$ does not have a universal spectrum. Indeed, if $S$ were a universal spectrum, then $|S|=|G| /|T|$ and $S-S \subset \cap_{i j} Z_{T_{i j}^{\prime}} \cup\{0\}$ would hold, and therefore $S-S \cap L-L=\{0\}$ 
would follow. That is, $S+L$ would be a tiling of $\hat{G}$, which is a contradiction because $L$ is not a tile, as observed in the paragraph after the definition of $L$. It remains to show the existence of $T_{i j}^{\prime}$.

Consider all possible $\bmod 8$ differences $\mathbf{k}_{i}-\mathbf{k}_{j}$ of the rows of the integer matrix $8 K$. Let $K-K$ denote the matrix containing these differences as row vectors. Now, regard $K-K$ as a mod 24 matrix and modify the entries by +8 or +16 in such a way that each row becomes a tile in $\mathbb{Z}_{24}$, and also the $\bmod 3$ rank of the resulting matrix $P$ is 3. It will soon be apparent why these modifications are helpful in finding the sets $T_{i j}^{\prime}$. We remark that the existence of such modifications appears to be pure luck. We give a possible example below:

$$
K-K=\left(\begin{array}{cccccc}
0 & 0 & 2 & 4 & 4 & 6 \\
0 & 0 & 4 & 2 & 6 & 4 \\
0 & 0 & 4 & 6 & 2 & 4 \\
0 & 0 & 6 & 4 & 4 & 2 \\
0 & 2 & 1 & 6 & 4 & 5 \\
0 & 2 & 4 & 1 & 5 & 6 \\
0 & 2 & 5 & 4 & 6 & 1 \\
0 & 2 & 6 & 5 & 1 & 4 \\
0 & 4 & 1 & 5 & 3 & 7 \\
0 & 4 & 2 & 6 & 6 & 2 \\
0 & 4 & 3 & 1 & 7 & 5 \\
0 & 4 & 5 & 7 & 1 & 3 \\
0 & 4 & 6 & 2 & 2 & 6 \\
0 & 4 & 7 & 3 & 5 & 1 \\
0 & 6 & 2 & 3 & 7 & 4 \\
0 & 6 & 3 & 4 & 2 & 7 \\
0 & 6 & 4 & 7 & 3 & 2 \\
0 & 6 & 7 & 2 & 4 & 3
\end{array}\right) \quad \longrightarrow\left(\begin{array}{cccccc}
0 & 16 & 2 & 4 & 12 & 14 \\
0 & 16 & 12 & 2 & 14 & 4 \\
0 & 16 & 12 & 14 & 2 & 4 \\
0 & 16 & 14 & 12 & 4 & 2 \\
0 & 2 & 1 & 14 & 12 & 13 \\
0 & 2 & 12 & 1 & 13 & 14 \\
0 & 2 & 13 & 12 & 14 & 1 \\
0 & 2 & 14 & 13 & 1 & 12 \\
0 & 12 & 1 & 13 & 11 & 23 \\
0 & 12 & 2 & 22 & 14 & 10 \\
0 & 12 & 11 & 1 & 23 & 13 \\
0 & 12 & 13 & 23 & 1 & 11 \\
0 & 12 & 22 & 2 & 10 & 14 \\
0 & 12 & 23 & 11 & 13 & 1 \\
0 & 22 & 10 & 11 & 23 & 12 \\
0 & 22 & 11 & 12 & 10 & 23 \\
0 & 22 & 12 & 23 & 11 & 10 \\
0 & 22 & 23 & 10 & 12 & 11
\end{array}\right)=P
$$

It is easy to check that all required properties are fulfilled. In fact, each row of the modified matrix $P$ has tiling complement $C_{1}=\{0,3,6,9\}$ or $C_{2}=\{0,1,6,7\}$ in $\mathbb{Z}_{24}$, and regarding $P \bmod 3$ an easy Gaussian elimination shows that the $1^{\text {st }}, 2^{\text {nd }}$ and $4^{\text {th }}$ rows $\mathbf{p}_{1}, \mathbf{p}_{2}, \mathbf{p}_{4}$ generate the others.

Observe that the set $T$ above is defined in such a way that the rows coincide mod 3 with $\mathbf{p}_{1}, \mathbf{p}_{2}, \mathbf{p}_{4}$ (and, of course, the entries of $T$ coincide $\bmod 8$ with those of $T_{1}$ ).

Consider now an arbitrary row vector $\mathbf{v}_{i j}=\mathbf{l}_{i}-\mathbf{l}_{j}$. We will exhibit the existence of the required tiling complement $T_{i j}^{\prime}$. For the sake of clarity we follow the proof through a particular example: let $\mathbf{v}_{31}=\mathbf{l}_{3}-\mathbf{l}_{1}=(3,0,0)-(0,0,0)=(3,0,0)$. Take the corresponding row $\mathbf{k}_{i}-\mathbf{k}_{j}$ of $K-K$, i.e., $\mathbf{k}_{3}-\mathbf{k}_{1}=(0,2,4,1,5,6)$ in our particular case. Consider the corresponding row $\mathbf{p}_{i j}$ of the matrix $P$, i.e., $(0,2,12,1,13,14)$ in our case. We claim that there exists a mod 24 row vector $\mathbf{y}_{i j}$ which is a solution of the equation $\mathbf{y}_{i j} T=\mathbf{p}_{i j} \bmod$ 24. Clearly, a solution of the same equation $\bmod 3$ exists, as $\mathbf{p}_{i j}$ is in the linear span of the rows of $T \bmod 3$ (recall that $T$ was chosen in such a way that its rows generate every vector $\left.\mathbf{p}_{i j} \bmod 3\right)$. In our case the $\bmod 3$ solution is seen to be $(0,2,0)$. A solution of the same equation mod 8 is simply obtained by dividing each entry of $\mathbf{v}_{i j}$ by 3, i.e., in our 
case a $\bmod 8$ solution is $(3,0,0) / 3=(1,0,0)$. (This is because $\frac{1}{3} L T=8 K \bmod 8$.) Then, a solution $\mathbf{y}_{i j} \bmod 24$ can easily be obtained from the $\bmod 3$ and $\bmod 8$ solutions; in our example it is $\mathbf{y}_{31}=(9,8,0)$.

Given such $\mathbf{y}_{i j}$ we can define a homomorphism $\varphi_{i j}: \mathbb{Z}_{24}^{3} \rightarrow \mathbb{Z}_{24}$ by the formula $\varphi_{i j}(\mathbf{x}):=\left\langle\mathbf{y}_{i j}, \mathbf{x}\right\rangle$. This homomorphism takes the set $T$ to the elements of the row $\mathbf{p}_{i j}$ by construction, and this resulting set tiles $\mathbb{Z}_{24}$ with complement $C_{i j}:=C_{1}$ or $C_{i j}:=C_{2}$ also by construction. In our example, $\varphi_{31}(T)=(0,2,12,1,13,14)$, which tiles $\mathbb{Z}_{24}$ with complement $C_{31}:=C_{1}=\{0,3,6,9\}$. Finally, the desired tiling complement $T_{i j}^{\prime}$ is defined as the pre-image of $C_{i j}$ under $\varphi_{i j}$. Here we need to invoke an elementary result of Szegedy [16].

Lemma 6. Let $G$ be a finite Abelian group, $T \subseteq G$ and suppose that there exists a homomorphism $\varphi: G \rightarrow H$ such that $\varphi$ is injective on $T$ and $\varphi(T)$ is a tile in $H$. Then $T$ tiles also $G$, and a tiling complement is given by $\varphi^{-1}\left(T^{\prime}\right)$ where $T^{\prime}$ is a complement of $\varphi(T)$.

Thus, we define $T_{i j}^{\prime}:=\varphi^{-1}\left(C_{i j}\right)$. It remains to check that $\mathbf{v}_{i j} \notin Z_{T_{i j}^{\prime}}$. The point of the whole construction above is that we can now evaluate $\hat{\chi}_{T_{i j}^{\prime}}\left(\mathbf{v}_{i j}\right)$. Note that each homomorphism $\varphi_{i j}$ is easily seen to be surjective (indeed, a homomorphism $\varphi(x, y, z):=$ $\langle(a, b, c),(x, y, z)\rangle$ is not surjective if and only if $a, b, c$ are all even or all are divisible by 3 ; whereas our vectors are not of this type). Therefore every element in $\mathbb{Z}_{24}$ has $24^{2}$ pre-images in $\mathbb{Z}_{24}^{3}$. Observe that $3 \mathbf{y}_{i j}=\mathbf{v}_{i j} \bmod 24$, hence for any $\mathbf{x} \in T_{i j}^{\prime}$ we have $\left\langle\mathbf{v}_{i j}, \mathbf{x}\right\rangle \in 3 C_{i j}$. Let $\rho=(1+i) / \sqrt{2}$ denote the first $8^{\text {th }}$ root of unity. Then

$$
\hat{\chi}_{T_{i j}^{\prime}}\left(\mathbf{v}_{i j}\right)=\sum_{\mathbf{x} \in T_{i j}^{\prime}} e^{2 \pi i / 24\left\langle\mathbf{v}_{i j}, \mathbf{x}\right\rangle}=\sum_{\mathbf{x} \in T_{i j}^{\prime}} e^{2 \pi i / 24\left\langle 3 \mathbf{y}_{i j}, \mathbf{x}\right\rangle}=\sum_{\mathbf{x} \in T_{i j}^{\prime}} e^{2 \pi i / 8\left\langle\mathbf{y}_{i j}, \mathbf{x}\right\rangle}=24^{2} \sum_{k \in C_{i j}} \rho^{k} \neq 0 .
$$

The last sum is non-zero as $\rho^{0}+\rho^{3}+\rho^{6}+\rho^{9} \neq 0$ and $\rho^{0}+\rho^{1}+\rho^{6}+\rho^{7} \neq 0$.

Putting together Propositions 5 and 3, and Theorems 2 and 1 we obtain a 3-dimensional counterexample to Fuglede's "tile $\rightarrow$ spectral" conjecture:

Corollary 7. There exists an appropriate finite union of unit cubes in $\mathbb{R}^{3}$ which tiles the space but which is not spectral.

Remark 3. At present, all known counterexamples to Fuglede's conjecture (in either direction, and in any dimensions) have their origins in the existence of complex Hadamard matrices with certain properties. It is conceivable that a tile having no universal spectrum (or a spectral set having no universal tiling complement) can be exhibited in a 1 or 2 dimensional finite group without any reference to Hadamard matrices. By the results of this paper such an example would immediately lead to a counterexample to (the corresponding direction of) Fuglede's conjecture. The 1-dimensional case seems particularly interesting, as it is related to the number theoretical conjecture of Coven and Meyerowitz. In search of a tile without universal spectrum we have conducted some numerical experiments in several cyclic groups. The main difficulty is the lack of quick algorithms for deciding whether a set is a tile, and whether it has universal spectrum. Given the lack of such algorithms we were unable to search large groups exhaustively, but our "sporadic" tests indicate that such examples, if they exist at all, are to be found in cyclic groups of fairly large order. 


\section{Appendix}

Here we exhibit an example which shows that the sufficient condition given in Proposition 4 is not necessary for the existence of a universal spectrum. This example was found earlier in [2], but there the authors could not decide whether the set given below has a universal spectrum.

Proposition 8. In the group $G=\mathbb{Z}_{6}^{4}$ the set given by the columns

$$
T:=\left(\begin{array}{cccccc}
0 & 1 & 0 & 0 & 0 & 2 \\
0 & 0 & 1 & 0 & 0 & 2 \\
0 & 0 & 0 & 1 & 0 & 2 \\
0 & 0 & 0 & 0 & 1 & 2
\end{array}\right)
$$

does have a universal spectrum $U$, while there exists no $S \subset \hat{G}$ such that $|S|=6^{3}$ and $S-S \subset Z_{T}^{c}$.

To see that such $S$ cannot exist we use the argument that has appeared several times above (and, in fact, was the basis of the arguments in [7]). Observe that $T$ is spectral in $G$ with a possible spectrum

$$
L:=2\left(\begin{array}{cccc}
0 & 0 & 0 & 0 \\
0 & 1 & 1 & 2 \\
1 & 0 & 2 & 2 \\
1 & 2 & 0 & 1 \\
2 & 2 & 1 & 0 \\
2 & 1 & 2 & 1
\end{array}\right)
$$

Note that $L$ is contained in the subgroup of elements with even coordinates. This subgroup has $3^{4}$ elements, therefore $L$ does not tile this subgroup, and hence does not tile $\hat{G}$ either. Now, the existence of a proposed set $S$ would mean, by Proposition 4 , that $S$ is a universal tiling complement of $T$, which is a contradiction as $L$ does not tile $\hat{G}$.

To see that $T$ does have a universal spectrum $U$ is more tricky. In fact, $U$ can be defined as $U:=\left\{\left(u_{1}, u_{2}, u_{3}, u_{4}\right): u_{1}+u_{2}+u_{3}+u_{4}=0 \bmod 6\right\}$. Then $U-U=U$ and therefore we only need to show that $U \subset \cap_{j} Z_{T_{j}^{\prime}} \cup\{0\}$ where $T_{j}^{\prime}$ runs through all the tiling complements of $T$. Write $U=U_{0} \cup U_{1}$, where $U_{0}=U \cap Z_{T}$ and $U_{1}=U \cap Z_{T}^{c}$. It is trivial from the Fourier condition $Z_{T} \cup Z_{T_{j}^{\prime}}=G \backslash\{0\}$ that $U_{1} \subset \cap_{j} Z_{T_{j}^{\prime}} \cup\{0\}$. It is an easy calculation to show that $U_{0}$ consists of all coordinate permutations of the vector $\mathbf{v}=(2,2,4,4)$. By symmetry of $T$ it is enough to show that $\mathbf{v} \in \cap_{j} Z_{T_{j}^{\prime}}$. This, however, is non-trivial and, unfortunately, we do not have a neat "structural" proof of this fact. In fact, the first proof that we found was listing out all tiling complements $T_{j}^{\prime}$ by a computer search (which, in itself, is a nearly impossible task due to the large size of the group $\mathbb{Z}_{6}^{4}$, and the lack of known quick algorithms.) Below we present a proof that is easy (but tedious) to check by hand.

Consider any tiling complement $T^{\prime}$ of $T$. We need to show that $\mathbf{v} \in Z_{T^{\prime}}$. Let $P$ denote the following set:

$$
P=\left\{(3,4,4,4)^{\top},(4,3,4,4)^{\top},(4,4,3,4)^{\top},(4,4,4,3)^{\top}\right\} .
$$

We will show the following: 
Fact 1. If $\mathbf{t} \in T^{\prime}$ then there exists an $\mathbf{x} \in P$ such that $\mathbf{t}+\mathbf{x} \in T^{\prime}$.

Fact 2. If $\mathbf{t} \in T^{\prime}, \mathbf{x} \in P$ and $\mathbf{t}+\mathbf{x} \in T^{\prime}$, then $\mathbf{t}+2 \mathbf{x} \in T^{\prime}$.

Fact 3. If $\mathbf{t} \in T^{\prime}, \mathbf{x}, \mathbf{y} \in P$ and $\mathbf{t}+\mathbf{x} \in T^{\prime}$ and $\mathbf{t}+\mathbf{y} \in T^{\prime}$ then $\mathbf{x}=\mathbf{y}$.

Assuming these facts for the moment, we can conclude our argument easily. Indeed, the above statements imply that $T^{\prime}$ is a disjoint union of 36 6-cycles of the form $C_{j}=$ $\left\{\mathbf{t}_{j}, \mathbf{t}_{j}+\mathbf{x}_{j}, \mathbf{t}_{j}+2 \mathbf{x}_{j}, \mathbf{t}_{j}+3 \mathbf{x}_{j}, \mathbf{t}_{j}+4 \mathbf{x}_{j}, \mathbf{t}_{j}+5 \mathbf{x}_{j}\right\}$ where $\mathbf{x}_{j} \in P$. Therefore,

$$
\hat{\chi}_{T^{\prime}}(\mathbf{v})=\sum_{j=1}^{36}\left(e^{2 \pi i / 6\left\langle\mathbf{v}, \mathbf{t}_{j}\right\rangle} \sum_{k=0}^{5} e^{2 \pi i / 6\left\langle\mathbf{v}, k \mathbf{x}_{j}\right\rangle}\right)=0,
$$

because the inner sums are all easily seen to be zero for all $x_{j} \in P$.

To show Fact 1 is easy. We can assume without loss of generality that $\mathbf{t}=\mathbf{0}$. Let us now see how the tiling $T+T^{\prime}=\mathbb{Z}_{6}^{4}$ covers the point $(4,4,4,4)^{\top}$. In order to cover $(4,4,4,4)^{\top}$, the set $T^{\prime}$ must contain one of the following points:

$\left\{(4,4,4,4)^{\top},(3,4,4,4)^{\top},(4,3,4,4)^{\top},(4,4,3,4)^{\top},(4,4,4,3)^{\top},(2,2,2,2)^{\top}\right\}$. However, $(4,4,4,4)^{\top} \in T^{\prime}$ would mean that $T+T^{\prime}$ covers 0 twice, while $(2,2,2,2)^{\top} \in T^{\prime}$ would mean that $T+T^{\prime}$ covers $(2,2,2,2)^{\top}$ twice.

Fact 3 is also straightforward by contradiction. We can assume once again that $\mathbf{t}=\mathbf{0}$ and, without loss of generality, that $\mathbf{x}=(3,4,4,4)^{\top}, \mathbf{y}=(4,3,4,4)^{\top}$. Then, $\mathbf{t}+\mathbf{x}=\mathbf{x} \in T^{\prime}$ and $\mathbf{t}+\mathbf{y}=\mathbf{y} \in T^{\prime}$ would imply that $T+T^{\prime}$ covers $(4,4,4,4)^{\top}$ twice, a contradiction.

The proof of Fact 2 is generated by a computer algorithm, but can also be checked by hand. We argue by contradiction. Assume that $\mathbf{t}=\mathbf{0} \in T^{\prime}, \mathbf{x}=\mathbf{t}+\mathbf{x}=(3,4,4,4)^{\top} \in T^{\prime}$, and $2 \mathbf{x}=\mathbf{t}+2 \mathbf{x}=(0,2,2,2)^{\top} \notin T^{\prime}$. Then $(1,2,2,2)^{\top}$ must be covered by $T+T^{\prime}$, and there are three ways to do it:

1. $(1,1,2,2)^{\top} \in T^{\prime}$

2. $(1,2,1,2)^{\top} \in T^{\prime}$

3. $(1,2,2,1)^{\top} \in T^{\prime}$

By symmetry of all appearing sets with respect to the last three coordinates, it is enough to check one of these cases, say 1; i.e. assume that $(1,1,2,2)^{\top} \in T^{\prime}$. Then $(0,0,0,5)^{\top}$ must be covered by $T+T^{\prime}$, and there are three ways to do it.

1.a. $(0,5,0,5)^{\top} \in T^{\prime}$

1.b. $(0,0,5,5)^{\top} \in T^{\prime}$

1.c. $(0,0,0,4)^{\top} \in T^{\prime}$

All of these cases lead to contradiction in the following manner:

Case 1.a.

$(0,0,5,0)^{\top}$ can only be covered if $(0,0,4,0)^{\top} \in T^{\prime}$, and then 
$(2,2,1,2)^{\top}$ can only be covered if $(2,2,1,1)^{\top} \in T^{\prime}$, and then $(4,4,3,4)^{\top}$ can only be covered if $(4,3,3,4)^{\top} \in T^{\prime}$, and then $(2,1,1,2)^{\top}$ can only be covered if $(2,0,1,2)^{\top} \in T^{\prime}$, and then $(1,5,5,0)^{\top}$ can only be covered if $(1,5,5,0)^{\top} \in T^{\prime}$, and then $(0,5,5,1)^{\top}$ can only be covered if $(5,5,5,1)^{\top} \in T^{\prime}$, and then $(5,0,0,1)^{\top}$ can only be covered if $(4,0,0,1)^{\top} \in T^{\prime}$, and then $(3,1,0,2)^{\top}$ can only be covered if $(3,1,5,2)^{\top} \in T^{\prime}$, and then $(2,5,4,0)^{\top}$ can only be covered if $(0,3,2,4)^{\top} \in T^{\prime}$, and then $(4,1,0,2)^{\top}$ cannot be covered without covering some point twice.

Case 1.b.

$(0,5,0,0)^{\top}$ can only be covered if $(0,4,0,0)^{\top} \in T^{\prime}$, and then $(2,1,1,2)^{\top}$ can only be covered if $(2,1,0,2)^{\top} \in T^{\prime}$, and then $(2,2,1,2)^{\top}$ cannot be covered without covering some point twice.

Case 1.c.

$(2,2,2,1)^{\top}$ can only be covered if $(2,2,1,1)^{\top} \in T^{\prime}$, and then $(1,2,2,1)^{\top}$ can only be covered if $(0,2,2,1)^{\top} \in T^{\prime}$, and then $(1,2,1,2)^{\top}$ can only be covered if $(1,2,0,2)^{\top} \in T^{\prime}$, and then $(4,4,4,3)^{\top}$ can only be covered if $(4,3,4,3)^{\top} \in T^{\prime}$, and then $(5,0,0,5)^{\top}$ can only be covered if $(5,0,5,5)^{\top} \in T^{\prime}$, and then $(1,3,1,1)^{\top}$ can only be covered if $(1,3,1,0)^{\top} \in T^{\prime}$, and then $(5,1,0,5)^{\top}$ can only be covered if $(5,1,0,5)^{\top} \in T^{\prime}$, and then $(5,0,1,5)^{\top}$ can only be covered if $(5,5,1,5)^{\top} \in T^{\prime}$, and then $(0,5,1,0)^{\top}$ can only be covered if $(4,3,5,4)^{\top} \in T^{\prime}$, and then $(0,0,1,5)^{\top}$ cannot be covered without covering some point twice.

\section{References}

[1] E. M. Coven, A. Meyerowitz, Tiling the integers with translates of one finite set, J. Algebra, 212 (1999)(1), 161-174.

[2] B. Farkas, Sz. Gy. Révész, Tiles with no spectra in dimension 4, Alfréd Rényi Institute of Mathematics preprint series, 10 (2004).

[3] B. Fuglede, Commuting self-adjoint partial differential operators and a group theoretic problem, J. Functional Analysis, 16 (1974), 101-121.

[4] A. Iosevich, N. Katz, T. Tao, The Fuglede spectral conjecture holds for convex planar domains, Math. Res. Lett., 10 (2003)(5-6), 559-569.

[5] A. Iosevich, N. H. Katz, T. Tao, Convex bodies with a point of curvature do not have Fourier bases, Amer. J. Math., 123 (2001)(1), 115-120.

[6] M. N. Kolountzakis, Non-symmetric convex domains have no basis of exponentials, Illinois J. Math., 44 (2000)(3), 542-550.

[7] M. N. Kolountzakis, M. Matolcsi, Tiles with no spectra, Forum Math., to appear. 
[8] M. N. Kolountzakis, M. Matolcsi, Complex Hadamard matrices and the spectral set conjecture, in: Proceedings of the 7th International Conference on Harmonic Analysis and Partial Differential Equations, El Escorial, 2004 To appear.

[9] S. Konyagin, I. Laba, Spectra of certain types of polynomials and tiling of integers with translates of finite sets, J. Number Theory, 103 (2003)(2), 267-280.

[10] I. Laba, Fuglede's conjecture for a union of two intervals, Proc. Amer. Math. Soc., 129 (2001)(10), 2965-2972 (electronic).

[11] I. Laba, The spectral set conjecture and multiplicative properties of roots of polynomials, J. London Math. Soc. (2), 65 (2002)(3), 661-671.

[12] J. C. Lagarias, S. Szabó, Universal spectra and Tijdeman's conjecture on factorization of cyclic groups, J. Fourier Anal. Appl., 7 (2001)(1), 63-70.

[13] J. C. Lagarias, Y. Wang, Spectral sets and factorizations of finite abelian groups, J. Funct. Anal., 145 (1997)(1), 73-98.

[14] M. Matolcsi, Fuglede's conjecture fails in dimension 4, Proc. Amer. Math. Soc., 133 (2005) (10), 3021-3026 (electronic).

[15] S. Pedersen, Y. Wang, Universal spectra, universal tiling sets and the spectral set conjecture, Math. Scand., 88 (2001)(2), 246-256.

[16] M. Szegedy, Algorithms to tile the infinite grid with finite clusters, in: 39th Annual Symposium on Foundations of Computer Science, pp. 137-147, Palo Alto, CA, USA, 1998.

[17] T. Tao, Fuglede's conjecture is false in 5 and higher dimensions, Math. Res. Lett., 11 (2004)(2-3), 251-258. 